\title{
Demonstração da geração de ondas eletromagnéticas estacionárias em um cabo coaxial
}

\author{
Demonstration of the generation of stationary electromagnetic waves on a coaxial cable \\ Vinícius Mariani Lenart ${ }^{1}$, Romeu Miqueias Szmoski ${ }^{1}$, Rozane de Fátima Turchiello ${ }^{1}$, Sergio \\ Leonardo Gómez ${ }^{* 2 @] ~}$ \\ ${ }^{1}$ Universidade Tecnológica Federal do Paraná, Departamento de Física, Ponta Grossa, PR, Brazil. \\ ${ }^{2}$ Universidade Estadual de Ponta Grossa, Departamento de Física, Ponta Grossa, PR, Brazil.
}

Recebido em 04 de Maio de 2020. Revisado em 07 de Maio de 2020. Aceito em 18 de Junho de 2020.

\begin{abstract}
Os conceitos de ondas estacionárias e modos normais são dos mais fundamentais da física, tanto da mecânica clássica como da quântica e do eletromagnetismo. Demonstrações usuais da existência de modos normais de oscilação incluem quase que exclusivamente modelos mecânicos, como por exemplo, cordas vibrantes. Neste artigo propomos um experimento simples para a geração e observação de ondas eletromagnéticas estacionárias em um cabo coaxial.
\end{abstract}

Palavras-chave: onda estacionária, onda eletromagnética, impedância.

The concepts of stationary waves and normal modes are among the most important concepts of physics, from classical mechanics to quantum mechanics and electromagnetism. The usual demonstrations of the existence of the normal modes of oscillation consider just mechanical models as, for example, vibrating strings. This communication proposes a simple experiment for produzing and observing electromagnetic stationary waves in a coaxial cable.

Keywords: stationary electromagnetic wave, coaxial cable, impedance.

\section{Introdução}

Os conceitos de ondas estacionárias e modos normais são dos mais fundamentais da física, tanto da mecânica clássica como da quântica e do eletromagnetismo. Eles estão presentes em modelos físicos relacionados, por exemplo, aos modos normais de oscilação de uma rede cristalina, às vibrações moleculares, aos elétrons num metal (elétrons de Bloch) e às oscilações dos campos eletromagnéticos numa cavidade óptica que originam um feixe laser. Assim, o estudo envolvendo a fundamentação teórica e a experimentação em relação aos modos normais de oscilação de um sistema se torna fundamental para a formação de um estudante da área de exatas. Diversos trabalhos publicados na literatura exploraram esse tópico, implementando estratégias experimentais baseadas na montagem de dispositivos mecânicos. Dentre esses trabalhos podemos destacar a utilização de cordas [1], instrumentos musicais [2], diapasões [3], motores [4] e um computador 5]. Porém, até o presente momento, houve poucas tentativas de abordagem experimental aos modos normais baseados na geração de ondas eletromagnéticas estacionárias [6] e na medida da velocidade de propagação de ondas eletromagnéticas em um cabo coaxial [7]. Ondas eletromagnéticas podem ser geradas por oscilações de carga elétrica devido à aplicação de uma diferença de

*Endereço de correspondência: sgomez@uepg.br. potencial senoidal em uma linha de transmissão. Nesse caso, tanto a corrente elétrica quanto a tensão passam a exibir o mesmo comportamento ondulatório. A propagação de ondas em guias de ondas, um tipo de linha de transmissão, é geralmente abordada teoricamente num curso de eletromagnetismo, mas raramente é visualizado em um laboratório. A transmissão de sinais usando linhas de transmissão é um assunto extremamente importante tanto do ponto de vista da física básica quanto do ponto de vista tecnológico. O intuito desse trabalho é o de propor um experimento simples para a formação de ondas eletromagnéticas estacionárias estabelecidas em um cabo coaxial, mediante um gerador de sinais, e que podem ser comprovadas com a ajuda de um osciloscópio pelo comportamento ondulatório correspondente da tensão elétrica. Assim, pretendemos contribuir para a melhoria do ensino nos cursos de graduação destinado à formação do quadro de profissionais da área de exatas.

\section{Fundamentação Teórica}

\subsection{Rede infinita de impedâncias}

Uma linha de transmissão, dos quais o cabo coaxial é um exemplo, é qualquer estrutura que permita a transferência entre dois pontos de sinais e energia na forma de ondas eletromagnéticas. O cabo coaxial é composto de um fio condutor interno e uma malha condutora externa 


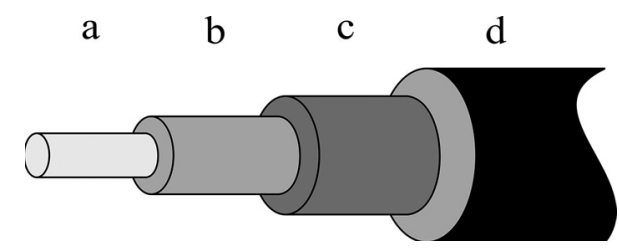

Figura 1: Esquema da estrutura de um cabo coaxial: a) fio condutor interno, b) dielétrico interno, c) malha condutora externa e d) isolante externo.

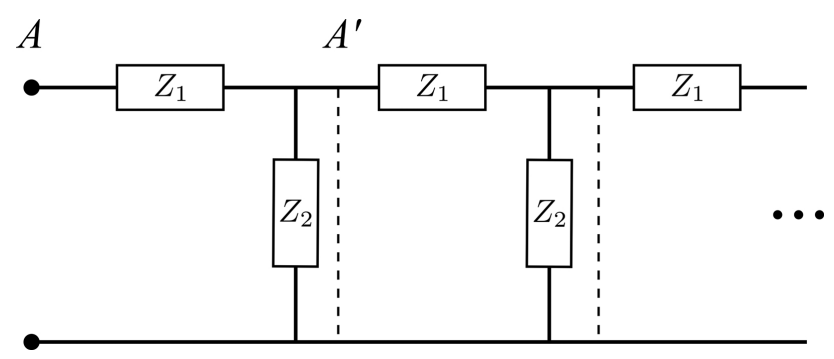

Figura 2: Rede linear semi-infinita de impedâncias, cujas unidades elementares incluem impedâncias $Z_{1}$ e $Z_{2}$.

separados por um meio dielétrico (Figura 1), o qual pode ser idealizado como um conjunto de parâmetros distribuídos, sendo estes indutância, capacitância e resistência dos dois condutores combinados e condutância associada ao material dielétrico. Para as frequências utilizadas nesse trabalho, consideraremos que a resistência e a condutância são desprezíveis (linha de transmissão sem perda). Assim, o cabo coaxial pode ser considerado como uma rede linear periódica de indutâncias e capacitâncias, ou seja, uma justaposição de unidades como as constituídas pelas regiões entre linhas paralelas tracejadas na Figura 2 8].

Consideremos a rede linear semi-infinita da Figura 2 formada por impedâncias de valor $Z_{1}$ localizados ao longo do condutor superior e impedâncias de valor $Z_{2}$ interligando ambos condutores. Supondo que à direita do ponto $A$ a rede semi-infinita possui um valor de impedância $Z_{e q}$, a parte da rede à direita de $A$ poderia ser substituída em sua totalidade por uma única impedância de valor $Z_{e q}$, mas sendo semi-infinita, também poderia ser substituída por uma impedância de valor $Z_{e q}$ com similar efeito somente a parte da rede semi-infinita à direita da linha tracejada por $A^{\prime}$ (Figura 3). Assim, podemos estabelecer a seguinte relação:

$$
Z_{e q}=Z_{1}+\frac{Z_{2} Z_{e q}}{Z_{2}+Z_{e q}}
$$

e portanto, o valor da impedância da rede semi-infinita é dada por:

$$
Z_{e q}=\frac{Z_{1}}{2}+\sqrt{\frac{Z_{1}^{2}}{4}+Z_{1} Z_{2}} .
$$

O resultado obtido nos mostra que a rede semi-infinita de impedâncias pode ser pensada como constituída da forma mostrada na Figura 4. Ou seja, o segundo termo

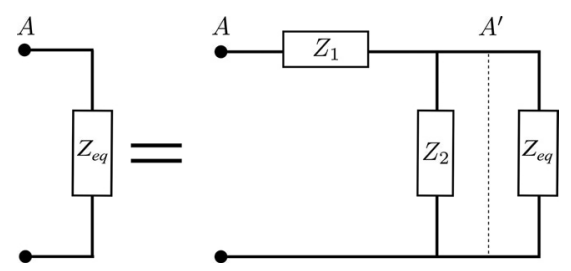

Figura 3: Uma rede semi-infinita de impedância $Z_{e q}$ pode ser substituída total ou parcialmente por uma única impedância de valor $Z_{e q}$.

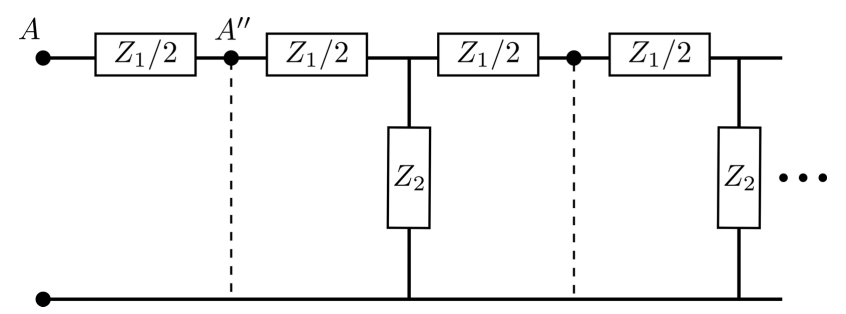

Figura 4: Forma alternativa de considerar a linha de transmissão semi-infinita.

de $Z_{e q}$ é a impedância da rede além da linha de traços que passa por $A^{\prime \prime}$ na Figura 4, a qual denominamos por $Z_{0}$ ou impedância característica da linha de transmissão. Supondo que $Z_{1}$ é uma reatância puramente indutiva dada por $Z_{1}=i \omega L$ e $Z_{2}$ é uma reatância puramente capacitiva dada por $Z_{2}=-i / \omega C$, onde $L$ e $C$ são os valores de indutância e capacitância dos elementos que formam a rede semi-infinita, respectivamente, $Z_{0}$ é dado por:

$$
Z_{0}=\sqrt{\frac{L}{C}-\frac{L^{2} \omega^{2}}{4}} .
$$

Para $0<\omega \leq \omega_{0}$, onde $\omega_{0}=2 / \sqrt{L C}$ é denominada frequência de corte, $Z_{0}$ é real, ou seja é puramente resistiva. Embora a rede seja composta por indutâncias e capacitâncias, a energia é "dissipada" na rede semiinfinita. Isso pode ser entendido pelo seguinte argumento: a energia fornecida pela fonte vai sendo armazenada nos suscessivos elementos indutivos e capacitivos na forma de campos magnéticos e elétricos, respectivamente. Portanto, a energia vai sendo transportada ao longo da rede semi-infinita. Assim, se finalizarmos um cabo coaxial de qualquer extensão com uma resistência no valor de $Z_{0}$, o cabo coaxial finito comportar-se-á como sendo um cabo semi-infinito.

\subsection{Onda progressiva numa linha de transmissão}

Com relação à rede semi-infinita de impedâncias vimos que a energia se propaga para sinais elétricos cuja frequência é menor que a frequência de corte $\omega_{0}$. Um cabo coaxial pode ser idealizado como sendo uma justaposição de seções de comprimento $\Delta x$, cada uma com uma indutância $L_{0}$ e uma capacitância $C_{0}$, ambas por unidade de compri- 
mento, na qual não há perdas. Assim podemos aplicar os resultados da seção anterior para o caso de indutâncias e capacitâncias de valor $L_{0} \Delta x$ e $C_{0} \Delta x$, respectivamente, tomando posteriormente o limite $\Delta x \rightarrow 0$. Portanto para um cabo coaxial ideal, $\omega_{0} \rightarrow \infty$ para $\Delta x \rightarrow 0$, podendo transmitir sinais eletromagnéticos na forma de ondas para virtualmente qualquer frequência, sendo que $Z_{0}$ é dado por:

$$
Z_{0}=\sqrt{\frac{L_{0}}{C_{0}}} .
$$

Para obtermos a equação de onda satisfeita no cabo coaxial, podemos considerar a diferença de potencial entre os pontos $x$ e $x+\Delta x$, onde $x$ é dado em relação a um extremo do cabo, ao longo do condutor interno do cabo (Fig. 5), é devida à indutância de valor $L_{0} \Delta x$ e é dada por 9 :

$$
V(x+\Delta x)-V(x)=-L_{0} \Delta x \frac{d I}{d t} .
$$

Dividindo a expressão anterior por $\Delta x$ e tomando o limite para $\Delta x \rightarrow 0$ obtemos:

$$
\frac{d V}{d x}=-L_{0} \frac{d I}{d t}
$$

Consideremos a corrente elétrica que circula pela seção no ponto $x$ do cabo. Se a corrente que sai do elemento em $x, I(x+\Delta x)$, é diferente da que entra na mesma região, $I(x)$, a diferença por unidade de tempo $I(x+\Delta x)-I(x)$ tem que estar sendo acumulada na capacitância da mesma região. Portanto podemos escrever:

$$
I(x+\Delta x)-I(x)=-C_{0} \Delta x \frac{d V}{d t} .
$$

Novamente dividindo ambos membros da equação anterior e tomando o limite para $\Delta x \rightarrow 0$ obtemos:

$$
\frac{d I}{d x}=-C_{0} \frac{d V}{d t} .
$$

As Equações 6 e 8 são denominadas de Equações da linha de transmissão ou Equações de telegrafia. Derivando a Eq. 6 em relação a $x$ e a Eq. 8 em relação ao tempo e combinando ambas expressões finais obtemos:

$$
\frac{d^{2} V}{d x^{2}}=L_{0} C_{0} \frac{d^{2} V}{d t^{2}} .
$$

Similarmente obtemos para $I$ :

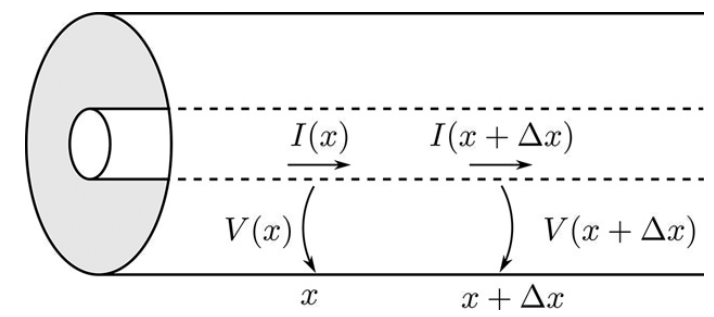

Figura 5: Correntes e voltagens observadas num cabo coaxial.

$$
\frac{d^{2} I}{d x^{2}}=L_{0} C_{0} \frac{d^{2} I}{d t^{2}}
$$

Assim, a voltagem e a corrente se propagam ao longo da linha de transmissão como uma onda com velocidade de fase dada por:

$$
v=1 / \sqrt{L_{0} C_{0}}
$$

\subsection{Ondas estacionárias}

Vimos nas seções anteriores que um cabo coaxial sustenta ondas progressivas de diferença de potencial e corrente elétrica praticamente para qualquer frequência, sendo possível a propagação em qualquer um dos sentidos. Também foi visto que uma rede semi-infinita pode ser reduzida na sua extensão se finalizamos ela com uma impedância cujo valor satisfaça a Eq. 3. Caso a impedância utilizada para finalizar a linha semi-infinita tenha um valor diferente, originar-se-á uma onda progressiva que se propagará no sentido contrário a qual interferirá com a onda propagando-se no sentido inicial. Este efeito é equivalente ao observado numa corda na qual é gerada uma onda num dos extremos. Quando a onda atingir o outro extremo, uma segunda onda é gerada nesse extremo, propagando-se no sentido contrário e interferindo com a onda incidente, com uma fase relativa à onda incidente dependente das condições no extremo final. Se este é fixo, a onda refletida é originada com uma diferença de fase igual a $\pi$ em relação à onda incidente, e se o extremo é livre, a fase relativa é $2 \pi$. Assim, a fase relativa da onda gerada no extremo do cabo coaxial dependerá da forma como este seja finalizado. $\mathrm{O}$ fato de haver um espectro contínuo de frequências de sinais que geram ondas estacionárias no cabo coaxial, nas condições de casamento de impedância entre o cabo e o gerador de frequências e para qualquer comprimento do cabo coaxial utilizado, permite fazer a analogia mecânica deste com a de uma corda de extensão infinita ou semi-infinita.

Considerando uma solução complexa das Equações 8 e 9, podemos escrever para a voltagem [10]:

$$
\begin{aligned}
\widetilde{V} & =\widetilde{V}^{+}+\widetilde{V}^{-} \\
& =\widetilde{V}_{0}^{+} e^{i(-k x+\omega t)}+\widetilde{V}_{0}^{-} e^{i(k x+\omega t)},
\end{aligned}
$$

onde o primeiro termo representa uma onda progressiva propagando-se no sentido positivo de $x$ e o segundo termo uma onda se propagando no sentido negativo de $x$. Na onda progressiva $v=\lambda f=\omega / k$. Expressão similar pode ser escrita para $\widetilde{I}$ :

$$
\widetilde{I}=\widetilde{I}_{0}^{+} e^{i(-k x+\omega t)}+\widetilde{I}_{0}^{-} e^{i(k x+\omega t)},
$$

lembrando que a tensão e a corrente efetivamente medidas são a parte real das expressões complexas $(V=\mathfrak{R}(\widetilde{V}), I=\mathfrak{R}(\widetilde{I}))$. A relação entre a voltagem e a corrente ao longo da linha de transmissão é: 


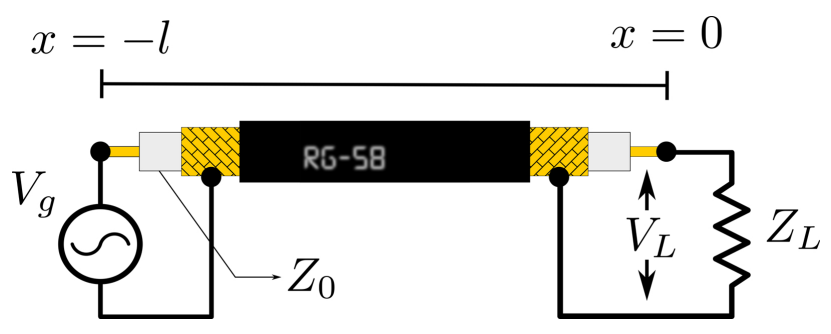

Figura 6: Esquema das voltagens presentes nos extremos da linha coaxial.

$$
\frac{\widetilde{V}(x)}{\widetilde{I}(x)}=Z_{0} .
$$

Consideremos $x=0$ no ponto da conexão do cabo coaxial com a carga. Assim, na conexão do cabo com o gerador teremos $x=-l$ (Figura 6$)$.

Em $x=0$ verifica-se também que:

$$
\begin{aligned}
Z_{L} & =\frac{\widetilde{V}(x=0)}{\widetilde{I}(x=0)} \\
& =\frac{\widetilde{V}_{0}^{+}+\widetilde{V}_{0}^{-}}{\widetilde{I}_{0}^{+}+\widetilde{I}_{0}^{-}} .
\end{aligned}
$$

Por outro lado, substituindo a expressão para $\widetilde{V}$ da Eq. 13 na Eq. 6. resolvendo para $\widetilde{I}$ e comparando com a Eq. 14 obtemos que:

$$
\frac{\widetilde{V}_{0}^{+}}{\widetilde{I}_{0}^{+}}=Z_{0}=-\frac{\widetilde{V}_{0}^{-}}{\widetilde{I}_{0}^{-}} .
$$

Assim, a Eq. 17 pode ser escrita como:

$$
Z_{L}=\frac{\widetilde{V}_{0}^{+}+\widetilde{V}_{0}^{-}}{\widetilde{V}_{0}^{+}-\widetilde{V}_{0}^{-}} Z_{0},
$$

ou resolvendo para $\widetilde{V}_{0}^{-}$:

$$
\widetilde{V}_{0}^{-}=\frac{Z_{L}-Z_{0}}{Z_{L}+Z_{0}} \widetilde{V}_{0}^{+}
$$

$\mathrm{O}$ coeficiente $\Gamma$ definido por:

$$
\Gamma \equiv \frac{Z_{L}-Z_{0}}{Z_{L}+Z_{0}}
$$

é chamado "coeficiente de reflexão" e em geral é complexo. No nosso caso, pelas condições impostas à impedância de carga $Z_{L}$, o coeficiente de reflexão é real. Da Eq. 21 vemos que $-1 \leq \Gamma \leq 1$, sendo -1 para uma impedância de carga nula, ou seja para um curto-circuito no extremo final da linha, 0 para uma impedância de carga igual à impedância característica (casamento de impedância) e em cujo caso não temos onda refletida, e 1 no caso de uma linha aberta no extremo final. Em termos de $\Gamma$, as ondas podem ser escritas como:

$$
\widetilde{V}=\widetilde{V}_{0}^{+}\left[e^{i(-k x+\omega t)}+\Gamma e^{i(k x+\omega t)}\right] .
$$

Em relação à analogia mecânica, a condição $Z_{L}=0$ $(\Gamma=-1)$ corresponde ao extremo final da corda sendo fixo, e o caso $Z_{L} \rightarrow \infty(\Gamma=1)$ ao extremo da corda sendo completamente livre. A superposição de ondas contrapropagantes de mesmo comprimento de onda e frequência leva à formação de ondas estacionárias na linha de transmissão. Lembrando que o sinal medido é a parte real da Eq. 22, obtemos:

$$
\begin{aligned}
|\widetilde{V}|^{2} & =\widetilde{V} \tilde{V}^{*} \\
& =\left|\widetilde{V}_{0}^{+}\right|^{2}\left[1+\Gamma^{2}+\Gamma e^{-i 2 k x}+\Gamma e^{i 2 k x}\right] \\
& =\left|\widetilde{V}_{0}^{+}\right|^{2}\left[1+\Gamma^{2}+2 \Gamma \cos (2 k x)\right]
\end{aligned}
$$

onde usamos a identidade $e^{i \alpha}+e^{-i \alpha}=2 \cos (\alpha)$. A Eq. 23 mostra que a amplitude da voltagem medida $V_{0}=$ $|\widetilde{V}|$ ao longo da linha de transmissão, quando nela se propagam ondas de número de onda $k$ e comprimento de onda $\lambda=\frac{2 \pi}{k}$, apresenta uma modulação espacial com número de onda $k^{\prime}=2 k$ e periodicidade espacial $\lambda^{\prime}=\lambda / 2$, tomando valores máximo e mínimo dados por $\left|\widetilde{V}_{0}^{+}\right|(1+|\Gamma|)$ e $\left|\widetilde{V}_{0}^{+}\right|(1-|\Gamma|)$, respectivamente. Assim, a diferença entre os valores máximos e mínimos da onda estacionária é dada por:

$$
\Delta V_{0}=2\left|\tilde{V}_{0}^{+}\right||\Gamma|
$$

A Figura 7 mostra três padrões espaciais para a amplitude da onda estacionária. Na Figura 7a é mostrada a amplitude da onda progressiva no sentido positivo de $x$, quando não há onda refletida devido ao casamento das impedâncias entre a linha e a carga em $x=0$. Nas Figura 7b e 7c são mostrados os padrões espaciais da amplitude no caso de um curto-circuito ou da conexão estar aberta em $x=0$, respectivamente. Das Figs. 7b e $7 c$ é possível observar que as curvas estão deslocadas em $\lambda / 4$ entre si.

Dado um cabo coaxial de comprimento $l$ com um curto-circuito em $x=0\left(Z_{L}=0 \Omega, \Gamma=-1\right)$, haverá um mínimo em $x=-l$ se $2 k l=2 \pi n$ para $n=1,2, \cdots$ e um máximo se $2 k l=(2 n-1) \pi$ para $n=1,2, \cdots$. Isso implica que as frequências das ondas progressivas em cada caso são:

$$
f_{n}= \begin{cases}\frac{v}{2 l} n & n=1,2, \cdots \text { (mín.) } \\ \frac{v}{4 l}(2 n-1) & n=1,2, \cdots \text { (máx.) }\end{cases}
$$

Caso em $x=0$ o extremo esteja aberto $\left(Z_{L} \rightarrow \infty, \Gamma=1\right)$, em $x=-l$ haverá um mínimo se $2 k l=(2 n-1) \pi$ para $n=1,2, \cdots$, e um máximo se $2 k l=2 \pi n$ para 
$n=1,2, \cdots$. Nesse caso as frequências das ondas progressivas correspondentes a cada caso são:

$$
f_{n}= \begin{cases}\frac{v}{2 l} n & n=1,2, \cdots \text { (máx.) } \\ \frac{v}{4 l}(2 n-1) & n=1,2, \cdots \text { (mín.) }\end{cases}
$$

Das Equações 25e 26 podemos observar que as posições dos máximos e dos mínimos encontram-se trocadas entre si para as condições do extremo final em curto-circuito e aberto, e que as frequências correspondentes encontramse espaçadas entre si no valor de:

$$
\Delta f=\frac{v}{2 l}
$$

\section{Montagem e Procedimento Experimental}

O arranjo experimental utilizado para a visualização das ondas estacionárias em um cabo coaxial compreende um gerador de sinais (Juntec, JDS6600), o qual entrega ondas com frequências de até $20 \mathrm{MHz}$, um osciloscópio de dois canais (Tektronix, TBS 1052B) e um cabo coaxial de 15, 69 m cuja impedância característica é $50 \Omega$ (RG-58). Um esquema do arranjo experimental é mostrado na Figura 8. Sendo as impedâncias de saída do gerador de tensão de $50 \Omega$ e de entrada do osciloscópio de $1 \mathrm{M} \Omega$, o uso do cabo coaxial de $50 \Omega$ permite que não exista uma
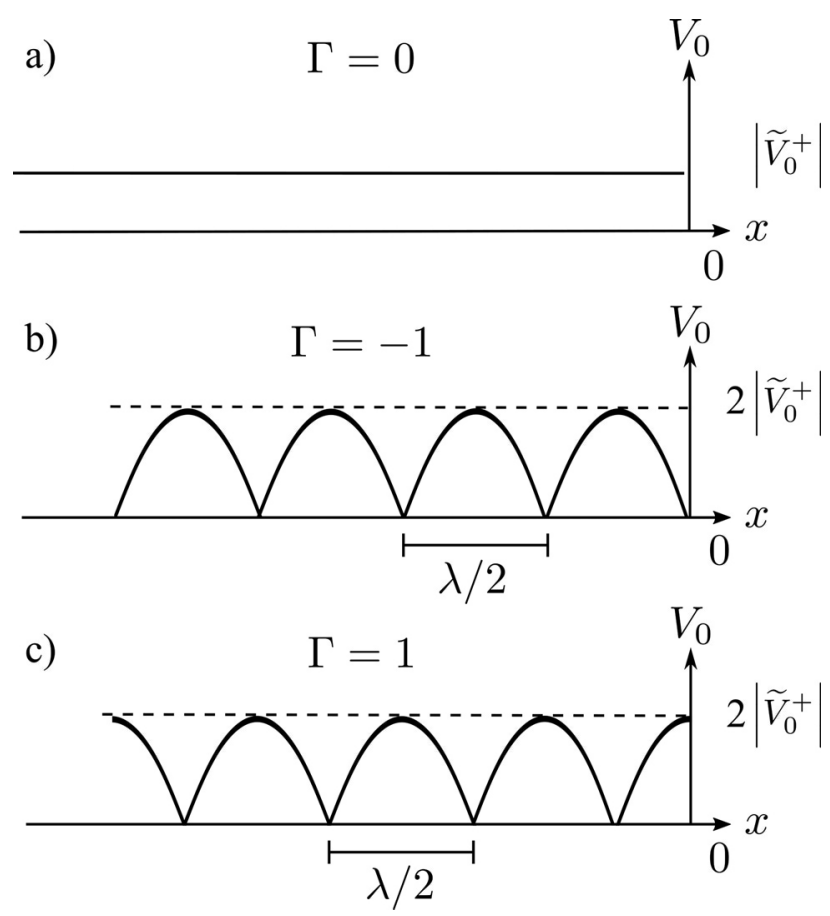

Figura 7: Amplitude $V_{0}$ da onda estacionária em função da posição $x$ ao longo do cabo coaxial para: a) casamento de impedância, b) curto-circuito, c) extremo aberto em $x=0$, sendo $\lambda^{\prime}=\lambda / 2$ a periodicidade espacial.

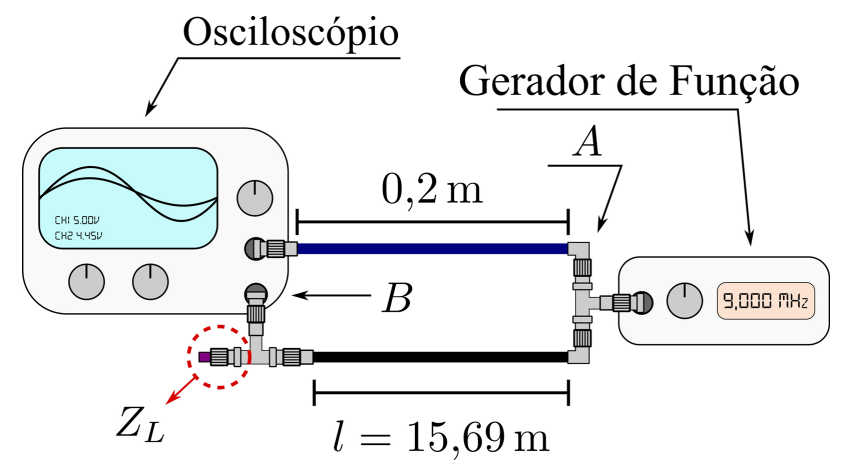

Figura 8: Esquema do arranjo experimental: gerador de sinais, osciloscópio e cabo coaxial.

reflexão no ponto de entrada da onda, a qual geraria outra onda que iria se superpor com a onda inicial e a onda refletida no outro extremo do cabo.

Com este arranjo foram determinadas a velocidade de fase das ondas progressivas e foi verificada a existência de ondas estacionárias no cabo. Para a determinação da velocidade de fase, um dos extremos do cabo coaxial finalizado com um conector BNC e denominado de $A$, foi conectado por meio de um conector BNC tipo $\mathrm{T}$ ao gerador de sinais e ao canal 1 do osciloscópio. O outro extremo do cabo coaxial, também finalizado com um conector BNC e denominado $B$, foi conectado por meio de um conector BNC tipo T a um terminal BNC de $50 \Omega$ e ao canal 2 do osciloscópio. Para a medida da velocidade de fase, é necessário o casamento de impedância no extremo $B$. Dessa forma, a onda injetada no ponto $A$ não gerará uma onda refletida no ponto $B$, e assim o cabo comportarse-á como um cabo semi-infinito. A velocidade de fase é dada pela relação $v=\frac{l}{\Delta t}$, onde $l$ é o comprimento do cabo e $\Delta t$ é o atraso do sinal no ponto $B$ em relação ao sinal medido em $A$. No nosso arranjo foi utilizado $0,2 \mathrm{~m}$ de um cabo coaxial de $50 \Omega$, ligando o gerador de função com o osciloscópio o qual teve que ser levado em conta na determinação da velocidade de fase na forma de uma diminuição no comprimento efetivo do cabo coaxial para $15,49 \mathrm{~m}$.

Para a verificação das ondas estacionárias o extremo $A$ foi ligado ao osciloscópio e ao gerador como no caso da medida da velocidade de fase, e o extremo $B$ estava em uma das três situações seguintes: aberto, em curtocircuito (unindo o condutor interno com a malha externa), ou ter um terminal BNC de $50 \Omega$.

É importante resaltar um ponto chave sobre o arranjo experimental: a diferença de fase entre as ondas nos pontos $A$ e $B$ é dada por $\Delta \varphi=\omega \Delta t=2 \pi f l / v$ e sendo a velocidade de fase da ordem de $10^{8} \mathrm{~m} / \mathrm{s}$, para que seja observada uma diferença de fase mensurável, é necessário levar em conta a frequência utilizada e o comprimento do cabo coaxial: um comprimento muito curto do cabo pode exigir grandes frequências, enquanto que um cabo longo permitiria utilizar frequências baixas. 
Para a medida da velocidade de fase, foi capturada a forma de onda senoidal, produzida pelo gerador, nos pontos de injeção do sinal $(A)$ e no extremo oposto $(B)$, observando o atraso da última em relação à primeira. O procedimento adotado para a obtenção das ondas estacionárias foi o seguinte: foi configurado o gerador de sinais para fornecer uma onda senoidal mudando a frequência em passos de $2 \times 10^{2} \mathrm{kHz}$ e para cada frequência do gerador, foi medida a amplitude $V_{0}$ do sinal senoidal no ponto A. A leitura das informações pode ser feita manualmente ou automatizada 11].

\section{Resultados e Discussões}

\subsection{Velocidade de fase}

A Figura 9 mostra o resultado da medida simultânea dos sinais nos pontos $A$ e $B$ para a frequência de $5 \mathrm{MHz}$. A partir destes dados podemos determinar a velocidade de fase da onda que se propaga ao longo do cabo coaxial. Os valores obtidos da velocidade de fase para frequências de até $20 \mathrm{MHz}$ são mostradas na Figura 10.

A velocidade de fase nessa escala de frequências é aproximadamente constante para frequências acima de $2,5 \mathrm{MHz}$, com valor médio $\bar{v}=2,03 \times 10^{8} \mathrm{~m} / \mathrm{s}$, resultado este que está de acordo com o valor obtido anteriormente [7]. Do valor médio da velocidade de fase, o valor previsto de $\Delta f($ Eq. 27) é aproximadamente $6,5 \mathrm{MHz}$.

\subsection{Ondas estacionárias}

A Figura 11 mostra a amplitude do sinal medido no ponto $A$ para 3 configurações do extremo $B$ : aberto, em curtocircuito e com um terminal BNC de $50 \Omega$. Como pode ser observado, a amplitude da tensão medida apresenta uma variação com a frequência do gerador, e os valores das frequências correspondentes aos máximos e mínimos

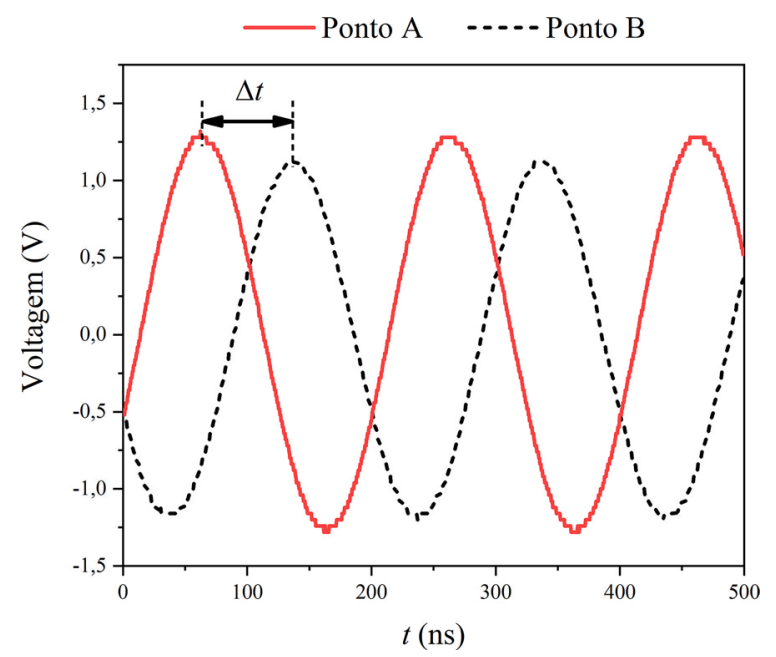

Figura 9: Sinais medidos em $A$ (linha contínua vermelha) e em $B$ (linha de traços preta) para a frequências de $5 \mathrm{MHz}$ do gerador. das amplitudes correspondentes às impedâncias de carga de $0 \Omega$ e $\infty$ estão trocadas entre si.

Os valores das frequências para a existência de um mínimo ou um máximo no ponto $A$ obtidos nesse trabalho para o cabo coaxial de 15,69 m são mostrados na Tabela 1.

É importante salientar que as frequências para a obtenção dos máximos e mínimos dependem estritamente do comprimento do cabo, ou seja um comprimento diferente

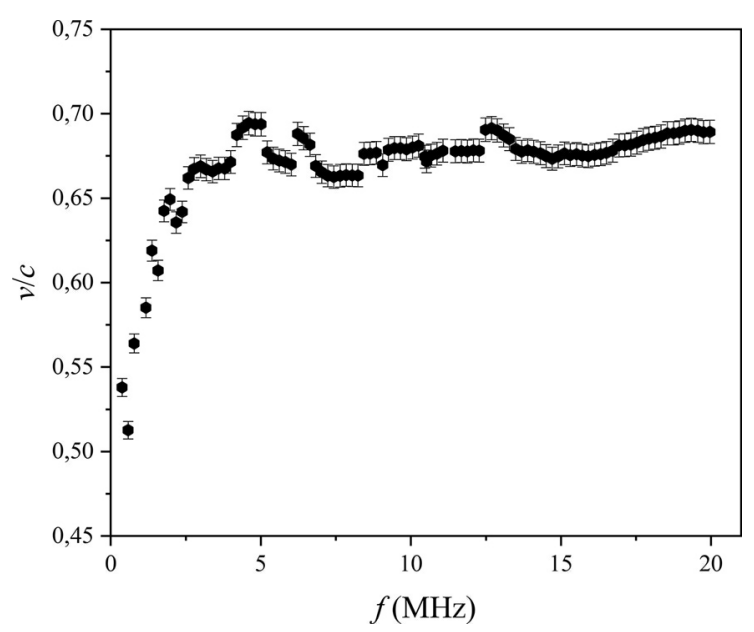

Figura 10: Velocidade de fase no cabo coaxial das ondas eletromagnéticas, em termos da velocidade de propagação no vácuo, para frequências de até $20 \mathrm{MHz}$.

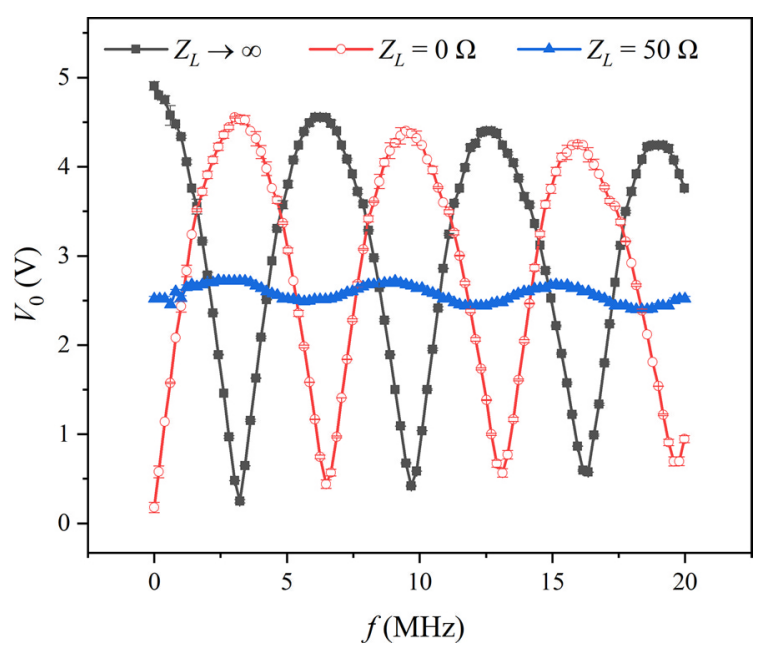

Figura 11: Gráfico da voltagem medida no ponto de injeção do sinal (ponto $A$ ) em função da frequência do gerador de sinais.

Tabela 1: Frequências, em unidades de $\mathrm{MHz}$, para a obtenção de um mínimo ou um máximo no ponto $A$, para o cabo RG-58 de $15,69 \mathrm{~m}$ de comprimento e impedâncias de carga de $0 \Omega \mathrm{e}$ $\infty$.

\begin{tabular}{ccccc}
\hline \multirow{2}{*}{$n$} & \multicolumn{2}{c}{ Máx. } & \multicolumn{2}{c}{ Mín. } \\
\cline { 2 - 5 } & $\Gamma=-1$ & $\Gamma=1$ & $\Gamma=-1$ & $\Gamma=1$ \\
\hline 1 & $3,0 \pm 0,2$ & $6,2 \pm 0,2$ & $6,5 \pm 0,2$ & $3,2 \pm 0,2$ \\
2 & $9,5 \pm 0,2$ & $12,5 \pm 0,2$ & $13,1 \pm 0,2$ & $9,7 \pm 0,2$ \\
3 & $16,0 \pm 0,2$ & $19,0 \pm 0,2$ & $19,6 \pm 0,2$ & $16,4 \pm 0,2$ \\
\hline
\end{tabular}


leva a frequências diferentes para os mesmos máximos e mínimos. A diferença de frequência entre máximos consecutivos e mínimos consecutivos têm uma excelente concordância com o valor previsto. No caso do uso do terminal BNC de $50 \Omega$, a curva de $V_{0}$ também apresenta um comportamento periódico com relação à frequência e de igual periodicidade. O motivo de não termos obtido uma linha reta no caso da impedância de carga de $50 \Omega$, assim como a variação com a frequência dos valores máximos e mínimos de $V_{0}$ pode ser devido ao fato de termos utilizado um modelo idealizado de uma linha coaxial de uso comercial, o qual não levou em conta uma possível componente resistiva no cabo coaxial.

\section{Conclusão}

Nesta comunicação apresentamos uma demonstração simples e didática da existência de ondas estacionárias de tensão em um cabo coaxial. Esta demonstração pode ser implementada facilmente num laboratório de física experimental básica em cursos da área de ciências exatas e engenharia, possibilitando a integração de conceitos básicos da mecânica e do eletromagnetismo. Assim, esperamos ter contribuído para a formação básica de nossos alunos de ciências exatas e engenharia.

\section{Agradecimentos}

Os autores agradecem à CAPES, Fundação Araucária, Universidade Tecnológica Federal do Paraná-Ponta Grossa e Universidade Estadual de Ponta Grossa pelo apoio financeiro. V. M. Lenart agradece também à CAPES pelo apoio financeiro (88882.316859/2019-01).

\section{Referências}

[1] C.A. Gomes e E. Lüdke, Rev. Bras. Ens. Fís.33, 3501 (2011).

[2] E.M. Santos, C. Molina, A.P.B. Tufaile, Rev. Bras. Ens. Fís. 35, 2507 (2013).

[3] A.J. Chiquito e A.C.A. Ramos, Rev. Bras. Ens. Fís. 27, 219 (2005).

[4] M.G. Souza, T.R. Cicogna e A.J. Chiquito, Rev. Bras. Ens. Fís. 29, 5 (2007).

[5] S.S.B. Jácome, F.F. de Medeiros, G. Corso e L.S. Lucena, Rev. Bras. Ens. Fís. 24, 214 (2002).

[6] C.E. Laburú, M.I. Nobre Ota, R.L. Oliveira Basso e C.J. Almeida, Cad. Bras. Ens. Fís. 21, 171 (2004).

[7] D.O.S. Gomes, W.S. Santos, A.C.F. Santos e C.E. Aguiar, Rev. Bras. Ens. Fís. 33, 3307 (2011).

[8] H.M. Nussenzveig, Curso de Física Básica (Ed. Blucher, São Paulo, 2007), v. 3, p. 222.

[9] R.P. Feynman, R.B. Leighton e M. Sands, The Feynman Lectures on Physics (Addison-Wesley, Boston, 1964), v. 2, p. 24.

[10] F.T. Ulaby, Eletromagnetismo para Engenheiros (Ed. Bookman, Porto Alegre, 2007).

[11] https://github.com/vmlenart/physics 